\title{
A cautionary tale for the physician: case report and review of food-dependent exercise-induced anaphylaxis
}

\author{
Kirk Evoy $^{1 *}$, Anita Wong ${ }^{2}$ and Stephen Simons ${ }^{3}$ \\ ${ }^{1}$ Department of Pharmacotherapy, University of Texas College of Pharmacy, USA \\ ${ }^{2}$ Department of Pharmacy, Jesse Brown VA Medical Center, USA \\ ${ }^{3}$ Department of Sports Medicine, Saint Joseph Regional Medical Center, USA
}

\begin{abstract}
Food-dependent exercise-induced anaphylaxis is a rare and underdiagnosed condition in which anaphylactic reactions present only in the setting of exercise within a few hours following (or rarely, immediately before) food intake. This case describes a 20-year old female with severe anaphylaxis developing during a run shortly after ingesting a meal containing shrimp. With acute epinephrine treatment, allergy workup, and education regarding separation of shrimp and exercise, this patient was able to resume her normal diet and exercise regimen without further harm. We hope this case raises awareness of this condition which may be overlooked by many clinicians due to lack of familiarity with the condition.
\end{abstract}

\section{Introduction}

Food-dependent exercise-induced anaphylaxis (FDEIA) is a rare subset of anaphylaxis that only occurs when a patient consumes the causative food (or in some cases non-specific food intake) within four to six hours prior to physical exercise. It may be triggered by a variety of foods, but most commonly wheat, shellfish, and peanuts, and is treated similarly to general anaphylaxis $[1,2]$. Though various case reports have described this phenomenon, FDEIA is rare and thought to be underdiagnosed as lack of familiarity with the condition likely results in many practitioners failing to include FDEIA in their differential diagnosis of patients presenting with anaphylaxis. With the publication of this case, we hope to raise awareness of physicians who may encounter such patients during exercise or athletic activities.

\section{Case report}

A 20-year old female presented to the emergency department (ED) with acute-onset anaphylaxis beginning during an evening run 1.5 hours post-ingestion of a dinner comprised of rice, shrimp, and lobster. Upon presentation, the patient was experiencing severe anaphylaxis which included head-to-toe hives followed by swelling of face, lips, tongue, and eyelids. Physical exam revealed mild tachycardia (108 beats per minute) and late expiratory wheezing but no shortness of breath and normal respiratory rate and blood pressure. Review of other systems was unimpressive. The patient presented within 1 hour of symptom onset and could not identify a potential cause. She reported no previous allergic reactions to food, medications, or exercise. She frequently consumed shrimp and other seafood and was a fairly regular runner, and neither had previously caused any symptoms. She had not consumed alcohol or cigarettes prior to the event and the only medication consumed was norgestimate/ethinyl estradiol oral contraceptive which she had been taking for some time. Her past medical history is notable only for seasonal allergic rhinitis, though this was mild and did not require chronic medications. In the ED intramuscular epinephrine was administered along with methylprednisolone, albuterol, diphenhydramine, and famotidine along with a liter of normal saline leading to rapid resolution of symptoms and she was discharged from the ED.

Post-discharge, the patient followed up as an outpatient for allergy testing. In allergy clinic, a percutaneous skin test was performed to identify potential reactions to various seafood allergens. Results (Table 1) displayed reactivity to shrimp, perch, clam, and oysters, of which the patient had only consumed shrimp prior to the incident. Additionally, radioallergosorbent tests (RAST - interpretation of this test is described in Table 2) were performed for shrimp and lobster on two blood samples. Shrimp was positive with scores of $0.65 \mathrm{kU} / \mathrm{L}$ and 1 $\mathrm{kU} / \mathrm{L}$ while lobster was negative with scores of $<0.35 \mathrm{kU} / \mathrm{L}$ and $0 \mathrm{kU} / \mathrm{L}$. Based on these results and the fact that the patient has regularly eaten shrimp and other forms of seafood without issue prior to this reaction, she was asked to return to clinic for an open oral challenge to shrimp to determine if exercise was required as a co-factor to trigger the reaction. Under physician supervision and with necessary medications

Table 1. Seafood percutaneous skin test results.

\begin{tabular}{|c|c|}
\hline Allergen & Result \\
\hline Clam & $3+$ \\
\hline Oyster & $2+$ \\
\hline Shrimp & $1+$ \\
\hline Perch & $2+$ \\
\hline Crab & 0 \\
\hline Lobster & 0 \\
\hline Scallops & 0 \\
\hline Other fish & 0 \\
\hline
\end{tabular}

Correspondence to: Kirk Evoy, PharmD, BCACP, BC-ADM, TTS, 7703 Floyd Curl Dr., San Antonio, Texas, 78229, USA, Email: evoy@uthscsa.edu

Key words: food-dependent exercise-induced anaphylaxis, anaphylaxis, allergy

Received: April 01, 2016; Accepted: April 18, 2016; Published: April 22, 2016 
Table 2. Radioallergosorbent test scoring chart.

\begin{tabular}{|c|c|}
\hline Concentration $(\mathbf{k U} / \mathbf{L})$ & Interpretation \\
\hline $0.00-0.35$ & Absent \\
\hline $0.35-0.70$ & Low \\
\hline $0.70-3.50$ & Moderate \\
\hline $3.50-17.5$ & High \\
\hline$>17.5$ & Very High \\
\hline
\end{tabular}

available to treat a subsequent allergic reaction, the patient ingested 0.5 shrimp, 1 shrimp, 2 shrimp, then ad libitum in 15 minute increments with no difficulty. The patient did not consent to a subsequent exercise challenge following ingestion of shrimp. However, given the timing of this reaction (i.e., ingesting shrimp without reaction followed by acute anaphylaxis beginning with exercise 1.5 hours after her meal), regular exercise of similar intensity without difficulty, history of tolerating shellfish on numerous occasions without symptoms and no recollection of previously exercising after eating shellfish, the positive skin and radioallergosorbent tests for shrimp allergy, and negative open oral challenge to shrimp, the patient was diagnosed with food-dependent exercise-induced anaphylaxis. She was informed that she may continue to ingest shrimp, however not to exercise for at least six hours after shellfish ingestion. She was prescribed epinephrine and advised to have it available during exercise. In following these recommendations, the patient has not experienced another episode in more than two years despite frequent seafood-containing meals, including shrimp and clams, and regular exercise.

\section{Discussion}

Exercise-induced anaphylaxis is a rare condition with an estimated prevalence of $0.048 \%$, or $5-15 \%$ of all anaphylactic cases $[3,4]$. Of these exercise-induced cases, $30-50 \%$ are thought to be FDEIA, signifying that the reaction only occurs if exercise takes place within hours of ingesting food (i.e., both the food and exercise are tolerated individually) [3-5]. FDEIA may develop at any age and reports differ with regards to differing probability among sexes. However, there is a higher prevalence among patients with a history of atopy [1].

In many cases, FDEIA occurs only after ingestion of specific foods, though some patients may experience symptoms following ingestion of food in general, without a specific trigger identified $[1,3,6]$. While numerous foods have been implicated in specific case reports (including fruits, vegetables, egg, mushrooms, garlic, meat, and rice, among others), the most common triggers of FDEIA include wheat, shellfish, and nuts.

Symptoms associated with FDEIA are similar to anaphylaxis from other causes, including pruritus, urticaria (typically manifesting as wheal and erythema and progressing to diffuse erythema), wheezing, dyspnea, abdominal pain, or nausea and vomiting $[4,6]$. Symptoms may quickly progress in severity to angioedema, laryngeal edema, loss of consciousness, hypotension or cardiovascular collapse and can be life-threatening, particularly if exercise persists. Approximately $80 \%$ of cases present with symptoms within 2 hours after ingestion of the causative food, precipitated by physical activity within this timeframe. This fact perhaps adds further to the rarity of the disease state, as some patients potentially susceptible to FDEIA may never exercise shortly after meals. Rarely, patients may experience symptoms when eating shortly after exercise. Additionally, due to the biphasic nature of anaphylaxis, patients may rarely experience late manifestations or second reaction hours after the exercise has ended [5].
The threshold of food or exercise needed to precipitate a reaction, as well as the symptom severity, displays great intra- and inter-patient variability, and thus symptoms are not always completely reproducible in a controlled setting $[2,6]$. Factors that may trigger FDEIA or alter the threshold to produce symptoms include the amount of food ingested; combination of foods eaten; exercise intensity, duration, or timing with relation to meals; patient specific factors such as fatigue, illness, or stress; menstruation; temperature extremes; season of the year; or concomitant ingestion of alcohol, aspirin, or NSAIDs $[1,6,7]$. Interestingly, aspirin has been noted to have a profound impact on FDEIA, lowering the threshold allergen dose necessary to trigger anaphylaxis (likely due to aspirin-induced alterations in gut permeability) and in some cases may even precipitate a reaction if taken with the causative food, even in the absence of exercise $[4,6]$.

A thorough clinical history eliciting all exposures and symptoms in the hours prior to presentation is the most important component in diagnosis. This history should include a detailed account of food intake and exercise preceding the event; history of past reactions to foods, exercise, or environment (e.g., insects, pollen, etc.); ingestion of prescription, non-prescription, and recreational drugs or alcohol; exposures to environmental extremes or physical or emotional stress; menstrual status in females; presence of acute respiratory or infectious conditions; and a detailed description of the symptoms, including their timing and progression $[1,2,5]$. Differential diagnoses that may be considered during initial workup include non-exercise-induced allergic reaction, exercise-induced anaphylaxis, cholinergic urticaria, exerciseinduced asthma, cold urticaria, mastocytosis, and angioedema, among others $[2,8]$.

In some cases, serum levels of mast cell mediators such as tryptase or histamine are assessed acutely, though their value is limited as tryptase levels have not been shown to be consistently elevated in patients presenting with anaphylaxis and histamine levels are elevated only for a short time [5]. Skin prick tests, in vitro serum food-specific IgE assays, or enzymatic immunoassays for allergen-specific IgE detection provide greater benefit, as these tests may result in identifying the causative allergen $[1,4,6]$. However, in the event of negative results, these tests lack the sensitivity to completely rule out potential allergens. Additionally, the results may be positive but unimpressive in magnitude and fall below the $95 \%$ positive predictive values for the diagnosis of food allergy [2]. If a potential causative allergen has not been identified, a broad panel of allergens may need to be assessed.

In cases where a potential food trigger has been identified via allergy testing, in-office provocation tests may lead to definitive diagnosis $[2,6,9]$. These may include food challenge alone, exercise alone (generally utilizing a treadmill and similar protocol to that used for the diagnosis of ischemic heart disease), and/or food ingestion followed by exercise. However, as many conditions may affect the FDEIA threshold, as discussed, challenge tests can only confirm diagnosis in approximately $70 \%$ of patients. Provocation tests also pose potential risk to the patient. If such tests are utilized, they should be performed under close supervision with equipment and medication readily available to treat a potential anaphylactic episode.

Though the pathophysiology of this condition is not entirely clear, it is thought to be an IgE-mediated hypersensitivity to food allergens, and several mechanisms have been proposed as to why these reactions occur only in the presence of exercise [3,4,6,10-13]. One hypothesis relates symptoms to decreased blood $\mathrm{pH}$ during exercise resulting in a lowered threshold for mast cell degranulation. To this regard, 
a limited number of case reports have shown that administration of sodium bicarbonate prior to physical activity may prevent symptoms in some patients $[12,13]$. However, as mild-moderate intensity exercise generally does not reduce physiologic $\mathrm{pH}$ but may produce FDEIA, decreased $\mathrm{pH}$ is not likely not the primary mechanism. Another theory associated with decreased threshold for mast cell degranulation points to the sympathetic system's redistribution of blood from the digestive tract to skeletal muscle and skin during exercise. As a result, food allergens that may have been tolerated by gut-specific mast cells activate less tolerant mast cells at the skin. Additionally, exercise affects gut permeability, potentially increasing the absorption of incompletely digested food which may trigger reactions outside of the gastrointestinal tract. Furthermore, aspirin has been shown to potentiate or trigger symptoms which may similarly be due to aspirin upregulating antigen uptake from the gastrointestinal tract. Finally, it has been suggested that exercise may activate tissue transglutaminase capable of aggregating proteins from the ingested food to produce large immunologic complexes leading to increased IgE binding. This has especially been linked to studies of wheat-dependent exercise-induced anaphylaxis.

Management of FDEIA can be broken down into acute treatment of the anaphylactic episode and long-term prophylaxis against future events [1]. In acute situations, first and foremost, exercise should be immediately ceased when symptoms develop to avoid further progression of the disease. Following cessation of exercise, acute treatment is similar to that of most other IgE-mediated allergic reactions with the use of epinephrine $(0.3 \mathrm{~mL}$ [0.3 mg] intramuscularly) as the mainstay of treatment. Histamine- 1 receptor antagonists (e.g., diphenhydramine), histamine-2 receptor antagonists (e.g., famotidine), corticosteroids, and albuterol may be provided as needed to further alleviate symptoms. However, these medications do not work as quickly, have not been shown to be life-saving in such an episode, and should only be used as adjunctive therapy to epinephrine, never as monotherapy for an anaphylactic event.

Long-term treatment for this condition includes patient education to avoid intake of causative foods prior to or immediately after exercise. If the causative food has been identified from subsequent workup, patients should not consume that food within three to six hours prior to or at least one hour after physical activity $[1,11]$. For non-specific cases in which a causative agent has not been identified, physical exercise should not be performed within three to six hours after consuming any food. However, some patients experience symptoms even with mild activity such as walking and in this case may need to avoid the causative food altogether [6]. Additionally, it is recommended for patients with FDEIA to avoid using NSAIDs, especially aspirin, as they have been shown to potentiate or even cause symptoms in the absence of exercise $[1,3,4,11]$. Finally, while no medications are generally recommended as chronic treatments to prevent FDEIA, it is imperative that patients are prescribed and carry an emergency automatic epinephrine injector during physical activity and that parents, athletic coaches, or others are trained in its use and present when the patient is resuming exercise [1]. In cases of refractory FDEIA that continues to occur despite avoidance of exercise following meals, some case reports have documented successful use of antihistamines, cromolyn, leukotriene receptor antagonists, sodium bicarbonate, or even omalizumab prophylactically, though there is no clear recommendation for these due to lack of clinical trials proving their efficacy $[1,5,14]$.

\section{Conclusion}

In this case, a young female who had previously tolerated shrimp and exercise alone, experienced severe anaphylaxis when ingesting shrimp shortly before her regular run. She was acutely treated with epinephrine as well as antihistamines, albuterol, and corticosteroids and experienced rapid symptom relief. Based on the results of a series of follow-up allergy tests, the timing of her symptom onset, and her negative open oral challenge to the probable food trigger, she was diagnosed with FDEIA, and with appropriate avoidance of exercise four-to-six hours after ingestion of shrimp or shellfish she has avoided additional anaphylactic episodes. This case highlights that, though rare and life-threatening, an accurate diagnosis of FDEIA and the causative food facilitate the safe independent return to exercise and prevent unnecessary diet restrictions.

\section{Funding/conflict of interest}

The authors received no funding for the development of this manuscript and have no conflicts of interest to report.

\section{References}

1. Kim CW, Figueroa A, Park CH, Kwak YS, Kim KB, et al. (2013) Combined effects of food and exercise on anaphylaxis. Nutr Res Pract 7: 347-351. [Crossref]

2. Du Toit G (2007) Food-dependent exercise-induced anaphylaxis in childhood. Pediatr Allergy Immunol 18: 455-463. [Crossref]

3. PovesiDascola C, Caffarelli C (2012) Exercise-induced anaphylaxis: A clinical view. Ital J Pediatr 38: 43. [Crossref]

4. Zogaj D, Ibranji A, Hoxha M (2014) Exercise-induced Anaphylaxis: the Role of Cofactors. Mater Sociomed 26: 401-404. [Crossref]

5. Bennett JR (2015) Anaphylaxis attributed to exercise: considerations for sports medicine specialists. Phys Sportsmed 43: 1-12. [Crossref]

6. Morita E, Kunie K, Matsuo H (2007) Food-dependent exercise-induced anaphylaxis. $J$ Dermatol Sci 47: 109-117. [Crossref]

7. Medveczky T (2014) A dangerous exercise lessons from food-dependent anaphylaxis for the physician. Am J Emerg Med 32: 1296. [Crossref]

8. Tewari A, Du Toit G, Lack G (2006) The difficulties of diagnosing food-dependen exercise-induced anaphylaxis in childhood -- a case study and review. Pediatr Allergy Immunol 17: 157-160.[Crossref]

9. Morita E, Chinuki Y, Takahashi H (2013) Recent advances of in vitro tests for the diagnosis of food-dependent exercise-induced anaphylaxis. J Dermatol Sci 71: 155159. [Crossref]

10. Mobayed HM, Ali Al-Nesf M (2014) Two cases of food-dependent exercise-induced anaphylaxis with different culprit foods. Ann Thorac Med 9: 42-44. [Crossref]

11. Del Giacco SR, Carlsen KH, Du Toit G (2012) Allergy and sports in children. Pediatr Allergy Immunol 23: 11-20. [Crossref]

12. AzofraGarcía J, Sastre Dominguez J, Olaguibel Rivera JM, Hernández de Rojas D EstupiñánSaltos M, et al. (1986) Exercise-induced anaphylaxis: inhibition with sodium bicarbonate. Allergy 41: 471. [Crossref]

13. Katsunuma T, Iikura Y, Akasawa A, Iwasaki A, Hashimoto K, et al. (1992) Wheatdependent exercise-induced anaphylaxis: inhibition by sodium bicarbonate. Ann Allergy 68: 184-188. [Crossref]

14. Bray SM, Fajt ML, Petrov AA (2012) Successful treatment of exercise-induced anaphylaxis with omalizumab. Ann Allergy Asthma Immunol 109: 281-282. [Crossref]

Copyright: (C2016 Evoy K. This is an open-access article distributed under the terms of the Creative Commons Attribution License, which permits unrestricted use, distribution, and reproduction in any medium, provided the original author and source are credited. 